\title{
Morphological and Topographic Anatomy of Sesamoid Bones of Human Thumb
}

\author{
R. Abisshek Balaji ${ }^{1}$, Apoorva ${ }^{1}$, V. V. Katti ${ }^{1}$, M. M. Pai ${ }^{2}$, B. V. Murlimanju ${ }^{2}$, L. V. Prabhu ${ }^{2}$ \\ 1 Final Year Medical Students, Kasturba Medical College, Mangalore, Manipal Academy of Higher Education, \\ Manipal, Karnataka, India \\ 2 Department of Anatomy, Kasturba Medical College, Mangalore, Manipal Academy of Higher Education, \\ Manipal, Karnataka, India
}

\author{
CORRESPONDING AUTHOR: \\ B. V. Murlimanju \\ Kasturba Medical College Mangalore \\ Mangalore, India \\ E-mail: flutemist@gmail.com
}

DOI:

10.32098/mltj.01.2020.19

LEVEL OF EVIDENCE: 4

\begin{abstract}
SUMMARY
Background. The objective of the present study was to study the morphology and topography of the sesamoid bones of the thumb in a sample south Indian population. Methods. The present study included 25 formalin fixed human cadaveric thumbs, the metacarpo-phalangeal and inter-phalangeal joints of the thumb were dissected. The number of sesamoid bones and the intrinsic muscles containing them were identified. The mean length, breadth and thickness of the sesamoid bones were measured before their removal from thumb by using the digital Vernier caliper.

Results. In $92 \%$ of cases, there were two sesamoid bones, observed at the metacarpo-phalangeal joint. The medial sesamoid bone was found inside the adductor pollicis muscle and lateral one was found either in the flexor pollicis brevis muscle $(64 \%)$ or abductor pollicis brevis muscle ( $28 \%$ ). The interphalangeal joint of the thumb in $96 \%$ of cases exhibited one sesamoid bone in its median plane, which was within the flexor pollicis longus tendon. The microscopic study confirmed the presence of only bone in all the sections. The mean length, width and thickness of the sesamoid bones at the metacarpo-phalangeal and inter-phalangeal joints of the thumb were $3 \pm 0.9 \mathrm{~mm}, 6.2$ $\pm 1.2 \mathrm{~mm}, 4.8 \pm 0.8 \mathrm{~mm}, 2.7 \pm 0.8 \mathrm{~mm}, 6.4 \pm 1.3 \mathrm{~mm}$ and $4.7 \pm 0.6 \mathrm{~mm}$ respectively. Conclusions. The present study has provided the morphometric data of the sesamoid bones and their topographical distribution in the intrinsic muscles of thumb. The data of the present study have implications in plastic surgery and will aid the operating hand surgeon and radiologist.
\end{abstract}

KEY WORDS

Intrinsic muscles; osteoarthritis; plastic surgery; tendons

\section{BACKGROUND}

Sesamoid bones are usually found within tendons in relation to the joint surfaces. They ossify after birth and are small nodular structures, which resemble a sesame seed. They may be completely ossified or may contain some cartilage. These sesamoid bones lack their outer covering, periosteum and fails to unite when fractured. Functionally the sesamoids, moderate the forces acting in a tendon. Earlier reports state that, there are as many as 42 sesamoid bones in the human body (1), patella being the largest. The sesamoid bones in hand are variable in topography and prevalence. They are incidental findings in routine hand X-ray films (2). The knowledge of sesamoid bones in the hand, is import- ant to understand the pathological conditions of the hand. The management of conditions like fracture of sesamoid (3), their inflammation (4) and sub-sesamoid joint degeneration (5), may require adequate knowledge about their morphology and topography. Often, these pathological conditions are misdiagnosed as trigger thumb (6), metacarpo-phalangeal joint arthritis (5) and chip fracture (7) by the hand surgeons (8). The post-traumatic sesamoiditis can cause disability of the thumb, which requires sesamoidectomy (9).

The detailed normal anatomy and morphological variants of sesamoid bones is essential to correlate clinically with the radiological finding (10). It was described that, human thumb contains two small sesamoid bones, which may be 
palpable at the base of the thumb (11). The medial and lateral sesamoid bones of thumb are believed to transmit force between the flexor pollicis brevis, adductor pollicis muscles and proximal phalanx of the thumb. The thumb sesamoids guide the flexor pollicis longus tendon as well. It was also reported that the range of movement of interphalangeal joint of thumb, depends on the presence or absence of the sesamoid bone (11). The sesamoid bones of thumb may vary in their morphology and topography across different population. Since the literature is scarce from our sample south Indian population, the present study was undertaken. The goal of the present study was to identify and locate the sesamoids in thumb, note the muscle containing them, record the number and estimate their length, width and thickness.

\section{MATERIALS AND METHODS}

The present study included 25 formalin fixed human cadaveric thumbs, which were utilized for teaching of the medical students. The study sample included thumbs (13 right and 12 left) from the 13 elderly (age ranged between 52-75 years), donated cadavers ( 9 male and 4 female) belonging to the south Indian population. The thumbs which exhibited pathological changes were excluded. Institutional ethic committee approval was taken before conduct of the study. It is also stated that this anatomical study meets the ethical international standards of this journal, which are essential as per the guidelines of Padulo et al. (12). The metacarpo-phalangeal and inter-phalangeal joints of the thumb were meticulously dissected and studied for the presence of sesamoid bones. The intrinsic muscles which contained the sesamoid bones were identified and noted. The dissections of thumbs were performed by two independent students, who are the first and fourth authors in this study. They have identified the sesamoid bones after the meticulous dissection and these were confirmed by the anatomy faculty, who are the second and third authors of this investigation. This was done to prevent the intra and inter observer errors. The sesamoid bones were then removed, washed and stored in formalin, with proper labeling (figure 1).

The mean length, breadth and thickness of the sesamoid bones were measured before removing them from the thumbs by using the digital Vernier caliper (Mitutoyo, Japan). To eliminate the observer bias, each measurement was taken independently by three authors and the average was considered. The morphometric data obtained in this study are represented as mean \pm SD and the dimensions are given in millimetre. The dimensions were analyzed statistically by using 'EZR software, version 1.38, 2019'. The statistical analysis of right and left side dimensions were done using the 'paired $t$ test'. The 'paired $t$ test' was also used to compare the medial and lateral sesamoid bones of the metacarpophalangeal joint. The gender wise statistical analysis of the dimensions were done by using 'independent samples $t$ test'. The 'p' value ( 2 tailed) less than 0.05 was considered as statistically significant.

To confirm the tissue present in these sesamoid bones, histological examination was performed. The sesamoid bones were sectioned transversely and longitudinally and observed under microscope and findings were noted. They were then decalcified using $20 \%$ nitric acid, paraffin sections prepared and stained using $\mathrm{H} \& \mathrm{E}$ stain. The sections were observed under high power of a trinocular camera upright microscope (Nikon, Nikon Corporation, Tokyo, Japan, model - Eclipse Ci-L). The findings were noted and images were captured using the software tool VUE.

\section{RESULTS}

The sesamoid bones were found in all of the thumb specimens $(100 \%$, figure 1$)$. In $92 \%$ of cases (23 thumbs), there were two sesamoid bones (figure 2 ), which were observed at the metacarpo-phalangeal joint. They were placed at the medial and lateral aspect of the metacarpo-phalangeal joint (figure 2). The medial sesamoid was within the adductor pollicis tendon and the lateral, either in the flexor pollicis brevis tendon ( $64 \%$ cases) or abductor pollicis brevis tendon ( $28 \%$ cases). However, two thumbs $(8 \%)$ had solitary sesamoid bone, which was observed in the adductor pollicis muscle. The inter-phalangeal joint of the thumb exhibited only one sesamoid bone (figure 3 ) at the medi-

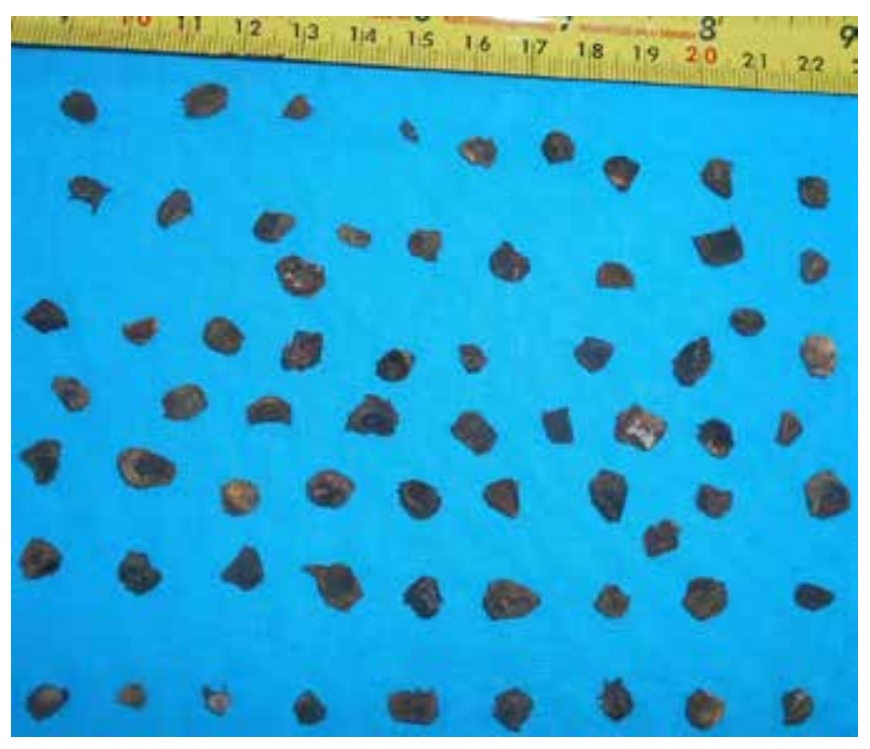

Figure 1. The sesamoid bones which are extracted from the cadaveric thumbs in this study. 


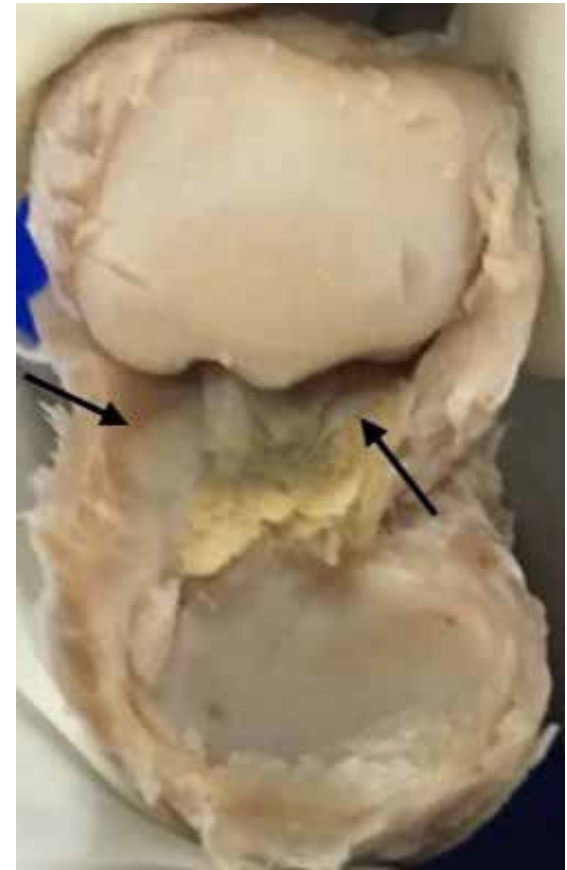

Figure 2. Metacarpophalangeal joint of the thumb showing two sesamoid bones (medial $\uparrow$, and lateral $\uparrow, 92 \%$ prevalence), found inside the tendons of adductor pollicis and abductor pollicis brevis/flexor pollicis brevis.

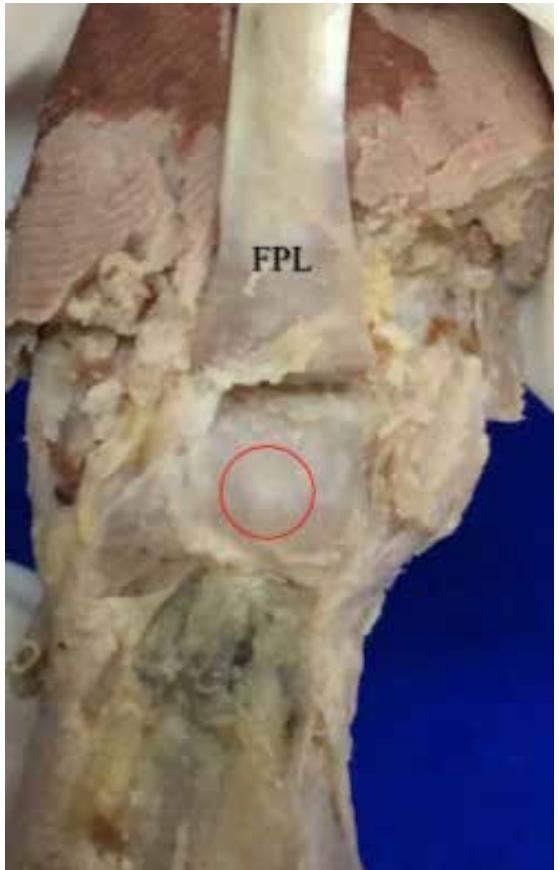

Figure 3. Palmar surface of the interphalangeal joint of the thumb showing a median sesamoid bone. This sesamoid bone (red circled) was attached to the flexor pollicis longus (FPL) tendon. an plane in $96 \%$ of cases (24 thumbs). However, there were two sesamoid bones at the interphalangeal joint in one of the thumbs $(4 \%)$ and was located on the flexor pollicis longus tendon (figure 3). The sesamoid bones and the intrinsic tendons containing them in this study are schematically represented in figure 4. Unstained histologic sections revealed Haversian canals and Volkmann's canals (figure 5A). Microscopic study of H\&E stained slides done after decalcification of the bones exhibited lacunae containing osteocytes (figure 5B). All the sesamoids contained only bony tissue, there wasn't any sign of cartilaginous tissue. The muscles of thumb containing sesamoid bones and their frequency of number and percentage are represented in table I. Table I also represents the length, width and thickness of the sesamoid bones in each tendon. The comparison of the morphometric data of the sesamoid bones of both sides, at metacarpophalangeal and interphalangeal joints are represented in table II

Table I. Frequency and dimensions of the sesamoid bones of thumb by each tendon.

\begin{tabular}{lllll}
\hline tendon \& sesamoid bone & frequency & length & width & thickness \\
\hline adductor pollicis $(\mathrm{n}=25)$ & $100 \%$ & $3.2 \pm 0.9 \mathrm{~mm}$ & $6.3 \pm 1.2 \mathrm{~mm}$ & $4.9 \pm 1 \mathrm{~mm}$ \\
\hline flexor pollicis brevis $(\mathrm{n}=16)$ & $64 \%$ & $2.9 \pm 0.7 \mathrm{~mm}$ & $6.2 \pm 1.2 \mathrm{~mm}$ & $4.7 \pm 0.6 \mathrm{~mm}$ \\
\hline abductor pollicis brevis $(\mathrm{n}=7)$ & $28 \%$ & $2.6 \pm 1 \mathrm{~mm}$ & $6 \pm 1.3 \mathrm{~mm}$ & $4.7 \pm 0.4 \mathrm{~mm}$ \\
\hline flexor pollicis longus $(\mathrm{n}=26)$ & $100 \%$ & $2.7 \pm 0.8 \mathrm{~mm}$ & $6.4 \pm 1.3 \mathrm{~mm}$ & $4.7 \pm 0.6 \mathrm{~mm}$ \\
\hline
\end{tabular}

measurements are given in mean $\pm \mathrm{SD}$

Table II. Sidewise comparison of the morphometric data of the sesamoid bones of thumb at metacarpophalangeal ( $n=48$ ) and interphalangeal joints $(n=26)$.

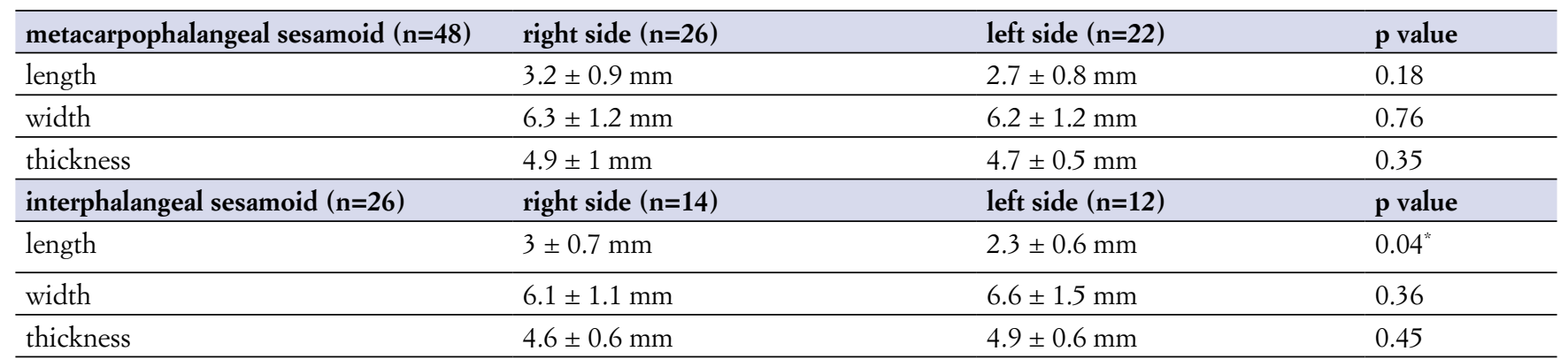

measurements are given in mean $\pm \mathrm{SD}$; statistical analysis - paired ' $\mathrm{t}$ ' test; significance, $\mathrm{p}<0.05$ 


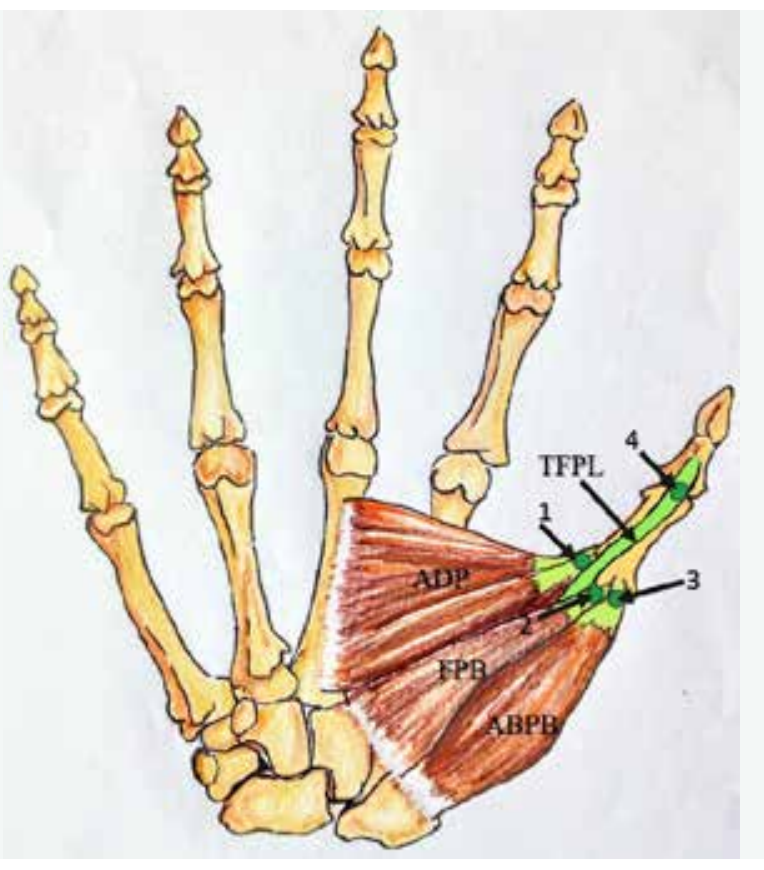

Figure 4. Schematic representation of the sesamoid bones and the intrinsic tendons containing them (ADP-adductor pollicis muscle; FPB-flexor pollicis brevis muscle; ABPB-abductor pollicis brevis muscle; TFPL-tendon of flexor pollicis longus muscle; 1-sesamoid bone in ADP; 2-sesamoid bone in FPB; 3-sesamoid bone in ABPB; 4-sesamoid bone in TFPL).

However these parameters were not statistically significant ( $>0.05)$ except the length of the interphalangeal sesamoid, which was longer on the right side $(\mathrm{p}=0.04)$. The gender wise comparison of the parameters (table III) also did not show any statistically significant differences $(\mathrm{p}>0.05)$.

The comparison of the morphometric data of the medial and lateral sesamoid bones (table IV) at metacarpophalangeal joint

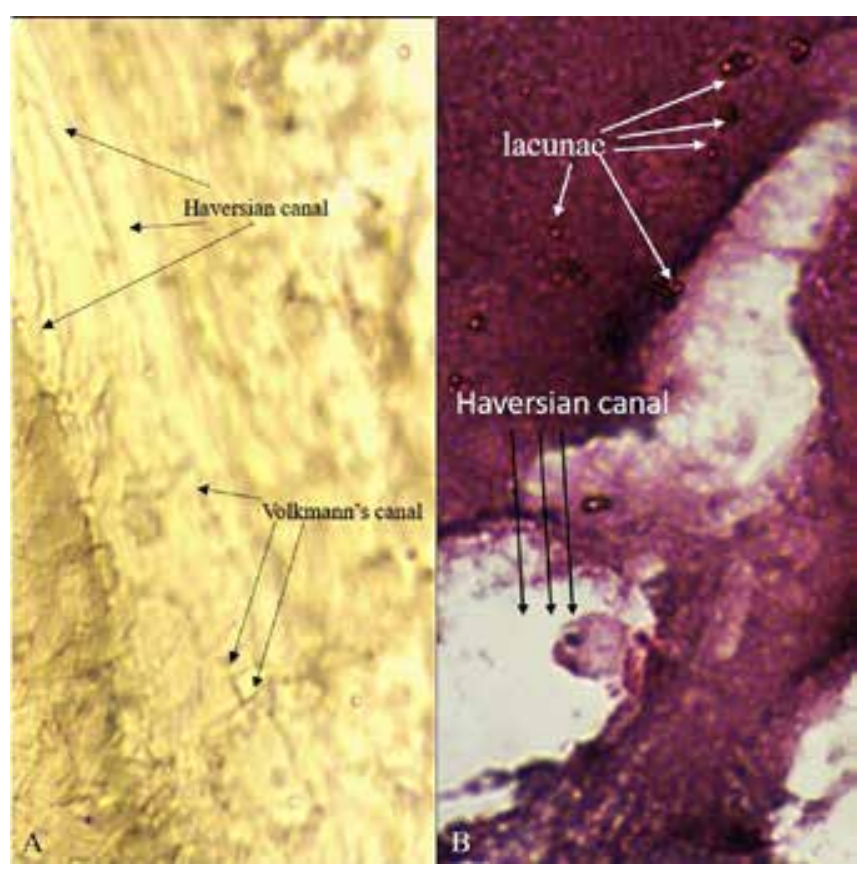

Figure 5A. Longitudinal section of the sesamoid bones showing Haversian canals and Volkmann's canals; 5B. High power view of the decalcified sesamoid bones showing Haversian canals and lacunae containing osteocytes (H \& E staining).

also didn't reveal statistically significant difference for any of the parameters measured $(p>0.05)$. The mean length, width and thickness of the sesamoid bones of metacarpo-phalangeal $(\mathrm{n}=48)$ and inter-phalangeal $(\mathrm{n}=26)$ joints of thumb are represented in table $\mathbf{V}$. The sesamoid bones at the inter-phalangeal and metacarpo-phalangeal joint were compared but the observations were not statistically significant $(\mathrm{p}>0.05)$.

Table III. Genderwise comparison of the morphometric data of the sesamoid bones of thumb at metacarpophalangeal $(n=48)$ and interphalangeal joints $(n=26)$.

\begin{tabular}{llll}
\hline metacarpophalangeal sesamoid $(\mathbf{n}=\mathbf{4 8})$ & male $(\mathbf{n}=\mathbf{3 2})$ & female $(\mathbf{n}=\mathbf{1 6})$ & $\mathbf{p}$ value \\
\hline length & $3.1 \pm 0.9 \mathrm{~mm}$ & $2.9 \pm 0.6 \mathrm{~mm}$ & 0.51 \\
\hline width & $6.2 \pm 1.5 \mathrm{~mm}$ & $6.2 \pm 1.5 \mathrm{~mm}$ & 0.98 \\
\hline thickness & $4.9 \pm 0.8 \mathrm{~mm}$ & $4.7 \pm 0.4 \mathrm{~mm}$ & 0.32 \\
\hline interphalangeal sesamoid $(\mathbf{n = 2 6})$ & male $(\mathbf{n}=\mathbf{1 8})$ & female $(\mathbf{n}=\mathbf{8})$ & $\mathbf{p}$ value \\
\hline length & $2.8 \pm 0.6 \mathrm{~mm}$ & $2.4 \pm 0.5 \mathrm{~mm}$ & 0.23 \\
\hline width & $6.1 \pm 1.5 \mathrm{~mm}$ & $6.9 \pm 1.9 \mathrm{~mm}$ & 0.13 \\
\hline thickness & $4.7 \pm 0.3 \mathrm{~mm}$ & $4.8 \pm 0.5 \mathrm{~mm}$ & 0.81 \\
\hline
\end{tabular}

measurements are given in mean $\pm \mathrm{SD}$; statistical analysis - independent' $\mathrm{t}$ ' test; significance, $\mathrm{p}<0.05$ 
Table IV. Comparison of the morphometric data of the medial $(n=25)$ and lateral $(n=23)$ sesamoid bones of thumb at metacarpophalangeal joint $(n=48)$.

\begin{tabular}{llll}
\hline measurement & medial $(\mathbf{n = 2 5})$ & lateral $(\mathbf{n = 2 3})$ & p value \\
\hline length & $3.2 \pm 0.9 \mathrm{~mm}$ & $2.8 \pm 0.9 \mathrm{~mm}$ & 0.21 \\
\hline width & $6.3 \pm 1.2 \mathrm{~mm}$ & $6.2 \pm 1.2 \mathrm{~mm}$ & 0.62 \\
\hline thickness & $4.9 \pm 1 \mathrm{~mm}$ & $4.7 \pm 0.5 \mathrm{~mm}$ & 0.27 \\
\hline
\end{tabular}

measurements are given in mean $\pm \mathrm{SD}$; statistical analysis - paired ' $t$ ' test; significance, $\mathrm{p}<0.05$

\section{DISCUSSION}

'Sesamoid' was derived from the word 'sesame', an East Indian herbal plant which has oily edible seeds. The name is also due to its resemblance for the wheat grain. Sesamoids are described as 'key to a mystery; a way out of a difficulty' (1). These are common in the hand and foot, observed at the metacarpo-phalangeal and inter-phalangeal joints of the thumb, metacarpo-phalangeal joints of index and little fingers. However, they are rarely observed at the metacarpo-phalangeal joints of middle and ring fingers. In foot, they are usually located at the metatarso-phalangeal joint of the great toe. Patella, pisiform and fabella are well known sesamoid bones, which ossify in the tendons of quadriceps femoris, flexor carpi ulnaris and gastrocnemius muscles.

Sesamoid bones will minimize the friction between the articulating joint surfaces during movements. They alter the direction of pull of muscles and withstand pressure. Sesamoid bones are usually observed in the intrinsic muscles, which produce flexion of metacarpo-phalangeal and metatarso-phalangeal joints. They are covered with the cartilage, which connects into the synovial cavity. Sesamoid bones are of two types, type A and type B $(1,13)$. Patella and sesamoid bones found in relation to the thumb and great toe are type A. Type ' $A$ ' variety are found next to a joint, here the tendon of sesamoid bone is incorporated into the joint capsule (13). In other words, type ' $A$ ' sesamoid bone forms an extension of the joint articulation. Type 'B' variety are found where the tendons are angulated with the bony surfaces and they are separated from the underlying bone by a synovial bursa (13). In other words, type ' $\mathrm{B}$ ' is not associated with a synovial joint. The sesamoid bones of peroneus longus tendon are best examples for this variety (1). The type ' $\mathrm{A}$ ' sesamoid bone is more common in the upper limb and type 'B' sesamoid are more frequent in the lower extremity (13).

The thumb sesamoids can be fractured in sports injuries, like hyperextension of the thumb. Skillern (14) and Yammine (15) reported that fracture of metacarpo-phalangeal sesamoid of thumb was initially reported by the Germans. The
Table V. Morphometric data of the sesamoid bones of metacarpo-phalangeal $(n=48)$ and inter-phalangeal $(n=26)$ joints of thumb.

\begin{tabular}{llll}
\hline measurement & $\begin{array}{l}\text { metacarpo- } \\
\text { phalangeal joint }\end{array}$ & $\begin{array}{l}\text { interphalangeal } \\
\text { joint }\end{array}$ & $\begin{array}{l}\text { p } \\
\text { value }\end{array}$ \\
\hline length & $3 \pm 0.9 \mathrm{~mm}$ & $2.7 \pm 0.8 \mathrm{~mm}$ & 0.09 \\
\hline width & $6.2 \pm 1.2 \mathrm{~mm}$ & $6.4 \pm 1.3 \mathrm{~mm}$ & 0.76 \\
\hline thickness & $4.8 \pm 0.8 \mathrm{~mm}$ & $4.7 \pm 0.6 \mathrm{~mm}$ & 0.33 \\
\hline
\end{tabular}

measurements are given in mean \pm SD; statistical analysis - paired ' $t$ ' test; significance, $\mathrm{p}<0.05$

fracture of sesamoid may be confused with ruptures of ligament of metacarpo-phalangeal joint and volar plate (15). On few occasions, there may be a possibility of bipartite sesamoid (16). The sesamoids are often confused in the radiological films, as chip fractures (15). The sesamoid bones can cause triggering of thumb and may get pushed inside the metacarpo-phalangeal joint during thumb injuries, particularly in dislocation of the metacarpo-phalangeal joint $(15,17)$.

The present study has measured the dimensions of the sesamoid bones of thumb which is not being reported earlier in the literature. The dimensions provide volumetric information and may be helpful to understand the pathological conditions affecting these sesamoid bones. The dimensions of sesamoid bones of metacarpo-phalangeal and inter-phalangeal joints of thumb obtained in the present study, can be considered as novel in the scientific literature.

There are not many studies available about the sesamoid bones of the thumb specifically from our sample south Indian population. Literature search will reveal few radiological studies, but non anatomical. Archana et al. (18) observed sesamoid bones in the metacarpo-phalangeal joint of thumb in $97.5 \%$ cases in their radiological study. They were two, one medial and one lateral in $30.3 \%$ cases, and solitary in $66.3 \%$ cases. Our study observed two sesamoid bones at the metacarpo-phalangeal joint of thumb in $92 \%$ cases and solitary in $8 \%$ cases. We could observe the sesamoid bones at the metacarpo-phalangeal and interphalangeal joints of thumb in all our specimens (100\%). The $100 \%$ prevalence of sesamoid bones in the thumb was also reported previously in Israelian (2) and Chinese (8) populations. However, Japanese (7) could observe the thumb sesamoids only in $67 \%$ of their cases. It is possible that this population wise difference in the prevalence is due to ancestral variations as genetic and environmental factors play a role (2). The knowledge about the sesamoid bones of thumb are important to differentiate them from the foreign bodies in the thumb. The pathological conditions of sesamoid bone like 
their inflammation, degeneration and fracture, can cause pain (8). These conditions may initially require conservative management like prescribing analgesics and corticosteroid injections (19). On few occasions, sesamoidectomy may be required in refractory cases (8). The morphometric data and topography of the sesamoid bones are important for the clinical evaluation. The data are essential to calculate the sesamoid index, subsesamoid index and subsesamoid joint space 20. We believe that the present study has provided important morphological and topographic details about the sesamoid bones of the thumb in sample south Indian population. Since the movements of thumb like opposition and reposition are exclusively a human character, the data of the present study may also be important to understand the comparative anatomy of sesamoid bones of the thumb. The present study has provided morphological data about the topography, length, width, thickness of the sesamoid bones and the intrinsic muscles which contain them. The sesamoid bones were also examined histologically and the microscopic structure was studied. The data of the present study are enlightening to the radiologists and hand surgeons. The dimensions of sesamoid bones of thumb will help in the interpretation of hand radiological films. The anatomical measurements obtained in this study can be compared with the dimensions which are performed radiologically. The sesamoid bone of thumb should not be confused with the pathological growth. The dimensions are enlightening to the operating hand surgeon in making an accurate diagnosis. Prior knowledge about the dimension of sesamoid bone will help the procedures like arthroscopic sesamoidectomy. The data are also important to the morphologist and embryologist. The prevalence and dimensions of thumb sesamoids will help the clinician in understanding the joint biomechanics. The knowledge is essential to understand the development of sesamoid bones. The data will also help in the anthropological comparison of the sesamoid bones in various populations. We believe that the topographical and morphometric dimensions of the sesamoid bones of thumb, which are obtained in this study can be used as a morphological database for the sample south Indian population.

\section{ACKNOWLEDGEMENTS}

The authors thank Mr. Venkat Naidu, Ms. Kriti Jain, Mr. Hanumantha S, Ms. Navya Muthusamy and Ms. Shreya Matele, the final year medical students for the help offered.

\section{CONFLICT OF INTERESTS}

The authors declare that they have no conflict of interests.

\section{REFERENCES}

1. Mottershead S. Sesamoid bones and cartilages: An enquiry into their function. Clin Anat. 1988;1:59-62.

2. Amar E, Rozenblat Y, Chechik O. Sesamoid and accessory bones of the hand-an epidemiologic survey in a Mediterranean population. Clin Anat. 2011;24:183-187.

3. Furlanetto S, Rossoni L. Fractures of the sesamoid bones of the hand and foot. Minerva Ortop. 1968;19:789-793.

4. Brüser P. Pathology of the sesamoid bones of the hand. Handchir Mikrochir Plast Chir. 1994;26:302-306.

5. Trumble TE, Watson HK. Posttraumatic sesamoid arthritis of the metacarpophalangeal joint of the thumb. J Hand Surg Am. 1985;10:94-100.

6. Seybold EA, Warhold LG. Impingement of the flexor pollicis longus tendon by an enlarged radial sesamoid causing trigger thumb: a case report. J Hand Surg Am. 1996;21:619-620.

7. Seki Y, Hoshino Y, Kuroda H. Prevalence of sesamoid bones in the interphalangeal joint of the thumb and fingers: a radiographic study. Clin Anat. 2013;26:823-826.

8. Lam GYT, Chow ECS, Ng B, Ho CHG, Chan CW. Distribution of sesamoid bones in the hand - a study in the Chinese population. Journal of Orthopaedics, Trauma and Rehabilitation. 2017;23:45-48.

9. Connolly CM, Alsousou J, Hassan S, Williams NW. Don't avoid the sesamoids: fracture to the ulnar sesamoid of the first metacarpophalangeal joint. Emerg Med J. 2008;25:184.

10. Becciolini M, Bonacchi G. Fracture of the sesamoid bones of the thumb associated with volar plate injury: ultrasound diagnosis. J Ultrasound. 2015;18:395-398.

11. Gibeault JD, Saba P, Hoenecke H, Graham A. The sesamoids of the metacarpo-phalangeal joint of the thumb: an anatomical and clinical study. J Hand Surg Br. 1989;14:244-247.

12. Padulo J, Oliva F, Frizziero A, Maffulli N. Muscles, Ligaments and Tendons Journal - Basic principles and recommendations in clinical and field Science Research: 2016 Update. Muscles Ligaments Tendons J. 2016;6:1-5.

13. Resnick D, Niwayama G, Feingold ML. The sesamoid bones of the hands and feet: participators in arthritis. Radiology. 1977;123:57-62.

14. Skillern PG. On fractures of the sesamoid bones of the thumb. Ann Surg. 1915;62:297-301.

15. Yammine K. The prevalence of the sesamoid bones of the hand: a systematic review and meta-analysis. Clin Anat. 2014;27:1291-1303.

16. Mohler LR, Trumble TE. Disorders of the thumb sesamoids. Hand Clin. 2001;17:291-301.

17. Failla JM. Irreducible thumb interphalangeal joint dislocation due to a sesamoid and palmar plate: a case report. J Hand Surg Am. 1995;20:490-491.

18. Archana A, Sanakkayala S, Nalluri HB, Nagothu RS. Study of incidence and ossification of sesamoid bones in hands of south Indian population. Int J Anat Res. 2017;5:3505-3509.

19. Sims AL, Kurup HV. Painful sesamoid of the great toe. World J Orthop. 2014;5:146-150.

20. Glorioso M, Miozzi F, Sartori A, Di Brino M, Giovagnorio F. Morphometric evaluation of sesamoid bones of the first metacarpophalangeal joint with ultrasound. Clin Ter. 2013;164:e449-e452. 